\title{
Meta
}

Journal des traducteurs

Translators' Journal

\section{Liste des relecteurs 2011}

Volume 56, numéro 4, décembre 2011

URI : https://id.erudit.org/iderudit/1011248ar

DOI : https://doi.org/10.7202/1011248ar

Aller au sommaire du numéro

Éditeur(s)

Les Presses de l’Université de Montréal

ISSN

0026-0452 (imprimé)

1492-1421 (numérique)

Découvrir la revue

Citer ce document

(2011). Liste des relecteurs 2011. Meta, 56(4), 735-737.

https://doi.org/10.7202/1011248ar

Ce document est protégé par la loi sur le droit d'auteur. L'utilisation des services d'Érudit (y compris la reproduction) est assujettie à sa politique d'utilisation que vous pouvez consulter en ligne.

https://apropos.erudit.org/fr/usagers/politique-dutilisation/
Cet article est diffusé et préservé par Érudit.

Érudit est un consortium interuniversitaire sans but lucratif composé de l’Université de Montréal, l'Université Laval et l'Université du Québec à Montréal. Il a pour mission la promotion et la valorisation de la recherche. https://www.erudit.org/fr/ 


\section{Liste des relecteurs 2011}

Nous remercions chaleureusement tous ceux et celles qui ont donné de leur temps et qui ont contribué à la revue. Toute omission est involontaire de notre part, veuillez nous la signaler.

Nüzhet Berrin Aksoy (Atılım University)

Fabio Alves (Universidade Federal de Minas Gerais)

Bassey Antia (University of Maiduguri)

Maite Aragonés Lumeras (WIPO, Genève)

Fabienne Baider (Université de Chypre)

Michel Ballard (Université d'Artois)

Paul Bandia (Université Concordia)

Rocío Baños-Piñero (London Metropolitan University)

Salah Basalamah (Université d'Ottawa)

Allison Beeby (Universitat Autònoma de Barcelona)

Marie-Alice Belle (Université de Montréal)

Ann Bertels (Katholieke Universiteit Leuven)

Davy Bigot (Université Concordia)

Albert Branchadell (Universitat Autònoma de Barcelona)

Annie Brisset (Université d'Ottawa)

Joost Buysschaert (Université de Gand)

Ángela Campo (Université de Montréal)

Raymond Chakhachiro (University of Western Sydney)

Leo Chan (Lingnan University)

Frédéric Chaume Varela (Universitat Jaume I)

Martha Cheung (Hong Kong Baptist University)

Anne Condamines (Université de Toulouse 2 - Le Mirail)

Christian Cuxac (Université Paris 8 - Vincennes-Saint-Denis)

Loïc Depecker (Université Paris 3 - Sorbonne Nouvelle)

Joseph Dichy (Université Lumière - Lyon 2)

Gaétane Dostie (Université de Sherbrooke)

Barbara Dragsted (Copenhagen Business School)

Valérie Dullion (École de traduction et d'interprétation - Université de Genève)

Keiran Dunne (Kent State University)

Isabel Durán-Muñoz (Universidad de Málaga)

Álvaro Echeverri (Université de Montréal)

Marco Fiola (Ryerson University) 
Thierry Fontenelle (Union européenne) Judit Freixa (Universitat Pompeu Fabra) Nicolas Froeliger (Université Paris Diderot - Paris 7)

Chantal Gagnon (Université de Montréal)

Thierry Gallèpe (Université Michel de Montaigne - Bordeaux 3)

Isabel García Izquierdo (Universitat Jaume I)

Joaquín García Palacios (Universidad de Salamanca)

Edwin Gentzler (University of Massachusetts Amherst)

Anna Ghiglione (Université de Montréal)

Daniel Gile (Université Sorbonne Nouvelle - Paris 3)

Patrick Goethals (Université de Gand)

Dinda L. Gorlée (University of Bergen)

Sandra L. Halverson (University of Bergen)

Ghodrat Hassani (Allame Tabataba'i University)

Juliane House (University of Hamburg)

Amparo Hurtado Albir (Universitat Autònoma de Barcelona)

Miguel Ibáñez Rodríguez (Universidad de Valladolid)

Riitta Jääskeläinen (University of Joensuu)

Francisco Lafarga (Universitat de Barcelona)

Homa Lessan-Pezechki (Université de Provence)

Arnt Lykke Jakobsen (Copenhagen Business School)

Laurence Jay-Rayon (Université de Montréal)

Kathrine Ravn Jørgensen (Copenhagen Business School)

Francine Kaufmann (Bar-Ilan University)

Dorothy Kelly (Universidad de Granada)

Don Kiraly (Johannes-Gutenberg-Universität Mainz)

Christopher Larkosh (University of Massachusetts Dartmouth)

Gillian Lathey (Roehampton University)

Elisabeth Lavault-Olléon (Université Stendhal - Grenoble 3)

Sara Laviosa (University of Bari)

Elisabeth Le (University of Alberta)

Benoît Léger (Université Concordia)

$\mathrm{Mu}$ Lei (Guangdong University of Foreign Studies)

Kazem Lotfipour-Saedi (Université d'Ottawa)

Josep Marco (Universitat Jaume I)

Danièle Marcoux (Université Concordia)

Jean-Pierre Marquis (Université de Montréal)

Elizabeth Marshman (Université d'Ottawa)

Stella Mavroveli (Imperial College London)

Reine Meylaerts (Katholieke Universiteit Leuven)

Nolwenn Mingant (Université Paris 3 - Sorbonne Nouvelle)

Vicent Montalt i Resurrecció (Universitat Jaume I)

Aura Navarro (Université de Montréal)

Robert Neather (Hong Kong Baptist University)

Jian-Yun Nie (Université de Montréal)

Alexis Nouss (Cardiff University)

Sharon O'Brien (Dublin City University) 
Riitta Oittinen (University of Tampere)

Pilar Orero (Universitat Autònoma de Barcelona)

Emer O’Sullivan (Leuphana Universität Lüneburg)

Kemal Özmen (Université Hacettepe)

Presentación Padilla (Universidad de Granada)

Christine Pagnoulle (Université de Liège)

Despoina Papadopoulou (Aristotle University of Thessaloniki)

Luis Pegenaute (Universitat Pompeu Fabra)

Ioana Popa (Université Paris Ouest - Nanterre La Défense)

Encarnación Postigo Pinazo (Universidad de Málaga)

Anthony Pym (Universitat Rovira i Virgili)

Serge Quérin (Université de Montréal)

Rosa Rabadán (Universidad de León)

Najat Rahman (Université de Montréal)

Shyam Ranganathan (York University)

Kristin Reinke (Johannes-Gutenberg-Universität Mainz)

Isabel Reyes Rodríguez Ortiz (Universidad de Sevilla)

Antin Fougner Rydning (University of Oslo)

Julio César Santoyo (Universidad de León)

Christine Savinel (Université Paris 3 - Sorbonne Nouvelle)

Christina Schäffner (Aston University)

Candace Séguinot (York University)

Rakefet Sela-Sheffy (Tel Aviv University)

Francisco Sevillano Calero (Universidad de Alicante)

Miriam Shlesinger (Bar-Ilan University)

Maria Sidiropoulou (National and Kapodistrian University of Athens)

Nathan Sivin (University of Pennsylvania)

Madeleine Stratford (Université du Québec en Outaouais)

Şehnaz Tahir-Gürçağlar (Boğaziçi University)

Kayoko Takeda (Rikkyo University)

Jun Tang (Southeast University)

Carles Tebé Soriano (Universitat Pompeu Fabra)

Rita Temmerman (Erasmushogeschool Brussel)

Mohammad Thawabteh (Al-Quds University)

Sonja Tirkkonen-Condit (University of Joensuu)

Agnès Tutin (Université Stendhal - Grenoble 3)

Marc Van Campenhoudt (Institut supérieur de traducteurs et interprètes - Haute

École de Bruxelles)

Suzanne Villeneuve (Université du Québec à Montréal)

Klaas Willems (Université de Gand)

Geoffrey Williams (Université de Bretagne-Sud)

Malcolm Williams (Université d'Ottawa)

Zhijie Wu (Nanjing University of Science and Technology et University of California)

Anastasia Yannacopoulou (Universite Paris-Est Marne-la-Vallée)

Yinde Zhang (Université Paris 3 - Sorbonne Nouvelle) 